\title{
Extraction of stress and dislocation density using in-situ curvature measurements for AlGaN and GaN on silicon growth
}

\author{
Matthew Charles $^{1 *}$, Mrad Mrad ${ }^{1}$, Joël Kanyandekwe ${ }^{1}$, Victor Yon ${ }^{1}$, \\ 1. Univ. Grenoble Alpes, CEA, LETI, 38000 Grenoble, France \\ *Email: matthew.charles@cea.fr
}

Keywords : A3 Metalorganic vapor phase epitaxy; A1 Stresses; B1 Nitrides; B2 Semiconducting III-V materials;

\begin{abstract}
We have analyzed the in-situ measurements of bow and reflectance during growth of GaN on silicon layers for HEMT based devices, varying the quality of the layers by changing the AlN nucleation layer. By fitting the curves and applying the Stoney equation, we were able to extract stress profiles in the layers, and convert these into out of plane strain profiles. This allowed us to simulate X-Ray Diffraction profiles which matched well with measured data, confirming the validity of our data extraction. Finally, we see that we incorporate less stress with a higher dislocation density in the GaN layers, and so for a given set of growth conditions, we can infer the dislocation by looking at the relaxation rate.
\end{abstract}

\section{Introduction}

The growth of GaN on silicon is becoming important for the production of power electronic devices, with components being processed on $150 \mathrm{~mm}$ and $200 \mathrm{~mm}$ wafers [1] [2] [3]. However, due to the large thermal expansion mismatch between $\mathrm{GaN}$ and silicon, buffer layers must be grown between the AlN nucleation and the final GaN layers in order to properly control the bow of the wafer. As GaN has a higher thermal expansion coefficient than silicon, this means that a large amount of compressive stress must be incorporated into the layers in order to produce a wafer with a bow less than $50 \mu \mathrm{m}$ at room temperature, which is a requirement for processing the wafers in high quality cleanrooms [4]. The large lattice mismatch between silicon and AlN means that there is a high dislocation density, typically $>1 \times 10^{10} \mathrm{~cm}^{-2}$ in the AIN nucleation layers. This high dislocation density means that in the subsequent III-N layers, relaxation occurs and the compressive stress is reduced [5]. The objective of this study was to examine the measurements of the bow performed in-situ, and to extract data about the relaxation and the strain in the layers.

\section{Experimental Details}

The structures were grown by metal organic vapor phase epitaxy (MOVPE) using an AIXTRON Crius R200 close coupled showerhead tool, with growth on a single $200 \mathrm{~mm}$ diameter silicon wafer. The tool is fully automated, with wafer loading at $400^{\circ} \mathrm{C}$, and chlorine cleaning between each growth. Hydrogen was used as carrier gas for all layers, with tri-methyl gallium (TMGa), tri-methyl aluminum (TMAl) and ammonia $\left(\mathrm{NH}_{3}\right)$ used as the precursors for gallium, aluminum and nitrogen respectively.

The in-situ measurements were performed using an EpiCurve and EpiTwinTT from Laytec, allowing measurement of the wafer bow, the reflectance at wavelengths of $950 \mathrm{~nm}$ and $405 \mathrm{~nm}$ and corrected temperature readings. The AFM images were performed using a BRUKER Dimension platform, and the X-ray Diffraction (XRD) scans were performed on a Panalytical Xpert Pro tool in triple axis mode.

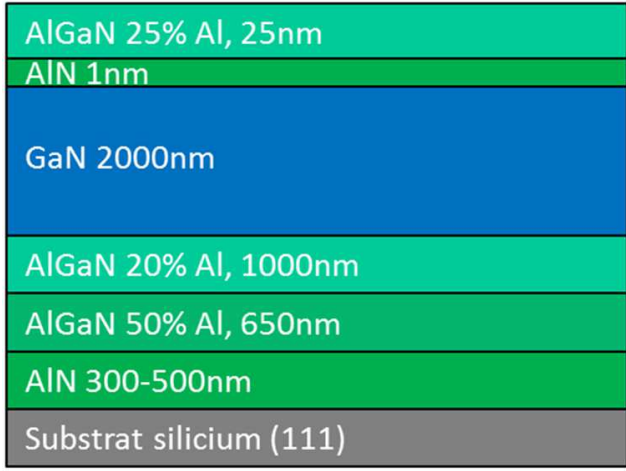

Figure 1: Schematic of grown structures

We have grown 4 structures on $1 \mathrm{~mm}$ thick $200 \mathrm{~mm}$ silicon wafers, with nominally on-axis ( $\left(\begin{array}{lll}1 & 1 & 1\end{array}\right)$ orientation and preparation of the wafers with a standard Radio Corporation of America (RCA) treatment [6]. The structure is shown in Figure 1 with AIN nucleation layer followed by $\mathrm{AlGaN}$ and $\mathrm{GaN}$ buffer layers. The AlGaN layers were grown with a high growth rate of $3.8 \mu \mathrm{m} / \mathrm{hr}$ to incorporate a high density of carbon while the GaN was composed of two parts. The first layer of $1.8 \mu \mathrm{m}$ $\theta$ f GaN[C]-was grown at high growth rate to give incerperate a high density of carbon concentration [7], 
and a "channel" layer of $200 \mathrm{~nm}$ with a lower carbon level in order to allow the generation of a 2-dimensional electron gas at the interface between the AlGaN and the $\mathrm{GaN}$. The barrier layer of $\mathrm{AlGaN}$ is a typical structure used for fabrication of high power $\mathrm{GaN}$ based devices [8]. For this study, we varied the quality of AIN nucleation layers, generating higher or lower dislocation density, by varying the growth conditions. The other layers were left unchanged throughout the experiments. Samples 1 to 4 were produced with gradually improving crystalline quality.

\section{Results and discussion}

Several steps of analysis are required to extract useful data from the curvature measurements. The initial measurements are performed at 6 points on the wafer, with $60^{\circ}$ separation, and then these are averaged. This average raw data is shown in Figure 2, with the different layers marked on the curve. Positive values correspond to concave wafers, and negative values to convex wafers. We see that the AlN layers take a long time to grow, and do not change significantly the wafer bow during the growth. The two AlGaN layers start to push the wafer towards a convex form, and then the GaN layer causes a large convex deformation. The wafer then reduces the convex bow as it cools down, becoming flatter, but it does not return to $0 \mathrm{~km}^{-1}$. This final value is not significant because the wafer is unloaded at $400^{\circ} \mathrm{C}$, and so we do not have a measurement of the wafer at room temperature.

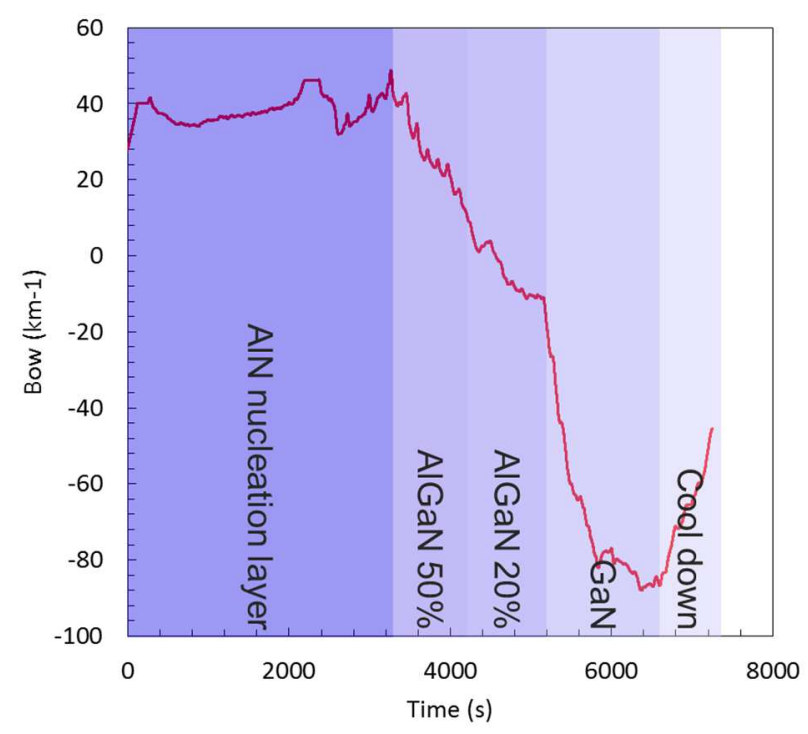

Figure 2: Curvature measurement for typical GaN on silicon growth, with individual layers shown. Positive bow is concave, and negative is convex.

The first step of the analysis of this curve is to examine the layers which cause significant changes in the bow, and then to convert the $\mathrm{x}$-axis from time to thickness. This is performed using the growth rates calculated from the 950 $\mathrm{nm}$ reflectance measurements, where the thickness for one oscillation:

$$
t=\frac{\lambda}{2 \eta}
$$

Where $\lambda$ is the wavelength reflected and $\eta$ is the refractive index of the material.

These calculated curves are shown in Figure 3 for all four samples. We see the evolution of bow for the three main layers, the two AlGaN layers and the GaN[C] layer. Although there is a clear evolution during the growth of these layers, the changes are not very smooth and there are also many peaks in the curves. These are caused by artefacts in the measurements, due to the difficulty of measurement of the bow through a very small view port.

In order to analyze these curves, we have fitted logarithmic curves to these data. These fitted curves are shown in Figure 4. Although these are not a perfect fit, they clearly follow the trends, and so are a good basis for the stress calculations.

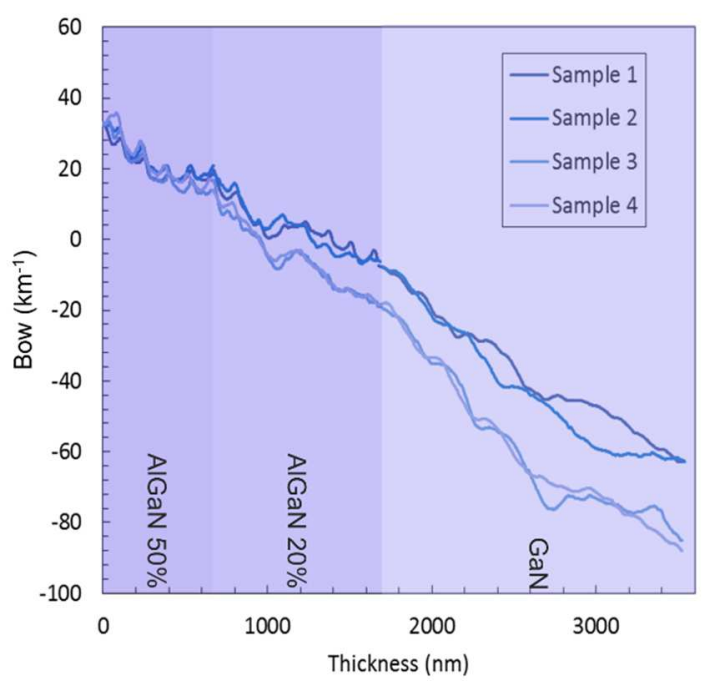

Figure 3: Evolution of bow in all 4 samples versus grown thickness

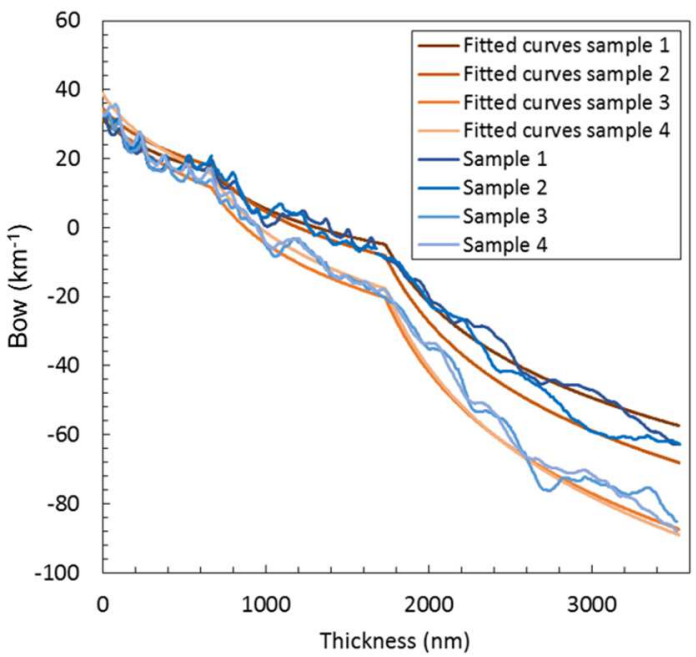


We can now apply the Stoney equation [9]:

$$
\sigma^{f}=\frac{E_{s} h_{s}^{2} \kappa}{6 h_{f}\left(1-v_{s}\right)}
$$

Where $\sigma_{\mathrm{f}}$ is the stress in the film, $\mathrm{E}_{\mathrm{s}}$ is the Young's Modulus of the substrate, $h_{s}$ is the substrate thickness, $\kappa$ is the wafer curvature, $h_{\mathrm{f}}$ is the film thickness and $v_{\mathrm{s}}$ is the Poisson's ratio of the substrate. In order to extract the stress, we need to know the change of curvature $\kappa$ for a given change in thickness of the film $h_{\mathrm{f}}$. We assume that the growth is uniform across the wafer, in terms of thickness, stress for all layers, and in terms of composition for the AlGaN layers. We also assume that the curvature is spherical in form.

We broke down the structure into $1 \mathrm{~nm}$ thick layers, each with a different stress, and then applied the Stoney equation, using $\Delta \kappa$ for the change in curvature rather than $\kappa$ and $\Delta \mathrm{h}_{\mathrm{f}}(=1 \mathrm{~nm})$ for the change in thickness of the film rather than $h_{f}$. Using the Young's Modulus for silicon of $160 \mathrm{GPa}$ [10], and the Poisson's ratio of 0.22 [10], we can calculate the stress in the film as a function of the thickness, as shown in Figure 5 for sample 1. We find peaks in the stress at the interface between each layer, as would be expected due to the abrupt change in lattice parameter. The stress subsequently reduces, as the layers relax. When we compare all 4 samples, as shown in red in Figure 6, we see that the higher quality layers have a higher peak stress, and the stress remains higher across the layers, even as all the layers relax.

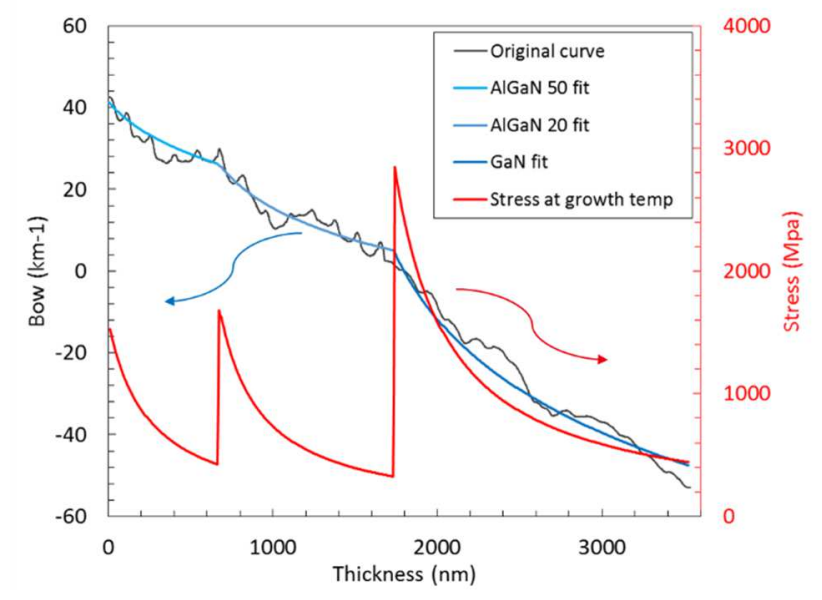

Figure 5: Stress in layers at growth temperature for sample 1

The next step was to take into account the stress added during the cool down to room temperature. This is done by comparing the lattice parameters of GaN [11], AlN [12] and silicon [13] at room temperature and growth temperature of $1050^{\circ} \mathrm{C}$, and assuming that the strain is applied by the silicon substrate on the III-N layers. The strain is then converted into a stress using the Young's Modulus of GaN [14] and AlN [15], and this stress is subtracted from the previously calculated values. The stress profiles at room temperature are shown in blue in
Figure 6. As the coefficient of thermal expansion (CTE) of $\mathrm{GaN}$ is larger than that of AlN, we see a greater shift for the $\mathrm{GaN}$ stresses than for the $\mathrm{AlGaN}$ layers, but otherwise there is a global shift towards tension with the reduction in temperature. We see from these data that we expect the GaN layers in sample 1 to be under greater tensile strain than those in sample 4, and this is confirmed by the AFM images in Figure 7, where we see that the pits generated in the surface of the $\mathrm{AlGaN}$ barrier layers are larger and more open in sample 1 than in sample 4. This is due to the additional tensile stress, which confirms qualitatively this prediction.

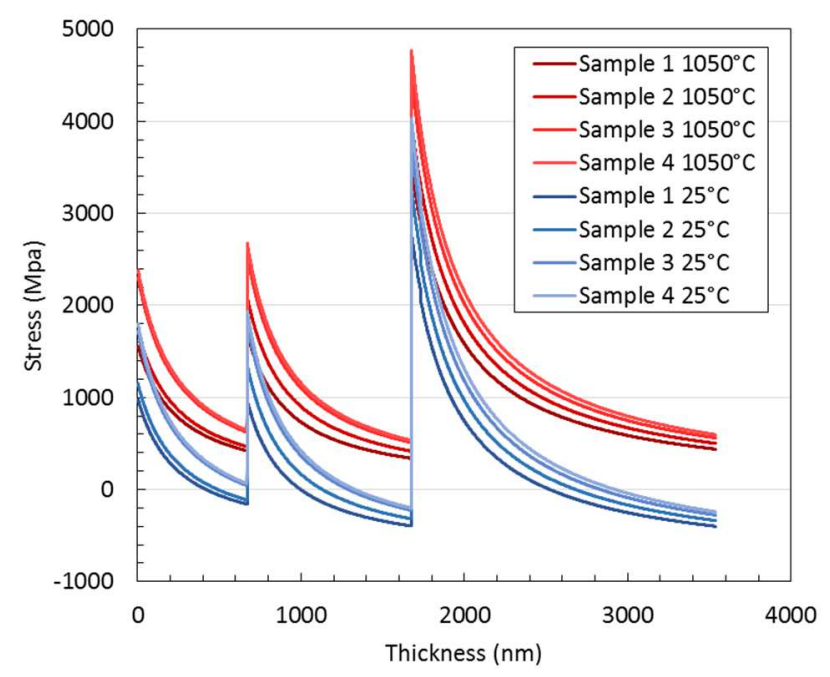

Figure 6: Stress in all 4 samples at growth temperature (red) and room temperature (blue)
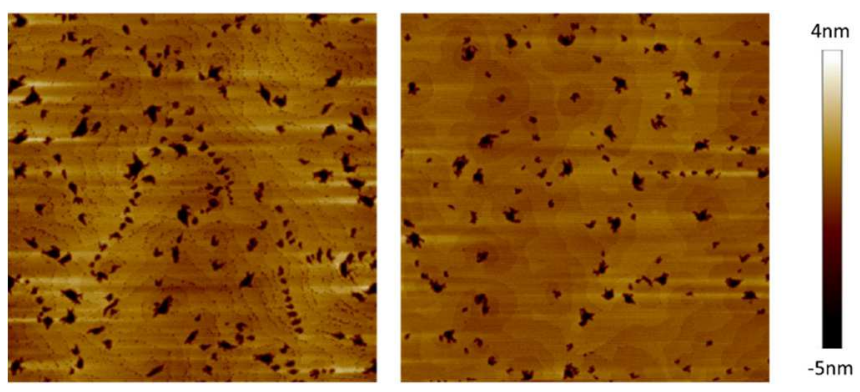

Figure 7: 2x2 $\mu m$ AFM images from sample 1 (left) and sample 4 (right)

In order to test more quantitatively the stress curves that we have modelled, we converted the in-plane stress values into in-plane strains, again using the Young's Modulus. Then, using the Poisson's ratio for GaN and AlN, we calculated the out of plane strain, and thus the planar spacing for the (002) planes. This allowed us to perform a simple simulation of the expected XRD spectra for a 2theta-omega curve, while not taking into account any scattering effects due to the grain size or dislocations. The effects of absorption in the layers are also neglected.

The results of these simulations are shown in Figure 8 in blue, with the measured data in red. We see a close match between the measured data and the simulated data, which could be considered surprising given the large number of 
assumptions in this study. In addition, we see that we achieve peak shapes which are similar to those of the real data. The GaN peaks in the real data are higher and narrower, but this may be due to the effects of absorption and the fact that after dislocation recombination, the last part of the layer is the highest quality, and so will have a higher peak intensity. However, we can see that the stress profiles that we have rebuilt from the in-situ curvature measurements match well with measured values. This therefore shows the effectiveness of this analysis. The differences between the $\mathbf{4}$ samples are greater in the simulated data than in the measured XRD data, and we suspect that this is due to the simple models used here for the simulated data. Higher resolution measurements with reduced artefacts could eliminate the need for fitted curves, and thus improve both the robustness and accuracy of these data, while more comprehensive XRD simulation would help confirm the matching of these curves.

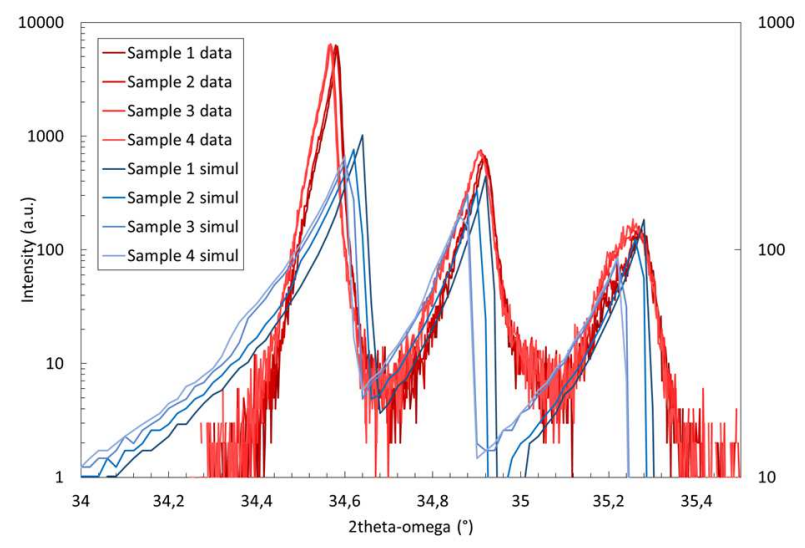

Figure 8: Measured XRD 2theta-omega curves on the (002) (red), and simulated curves from stress calculations (blue)

Finally, for the 4 samples described, we have compared the peak stress calculations with the dislocation density in the layers as measured by AFM. These data are shown in Figure 9, and we see that there is a good correlation between the two. This is to be expected, as relaxation typically occurs due to the recombination of dislocations, thus the higher their density, the lower the stress which is incorporated in the layers. This analysis implies that for given $\mathrm{GaN}$ growth conditions, we can infer the dislocation density of the layers by examining the peak stress as measured by in-situ devices.

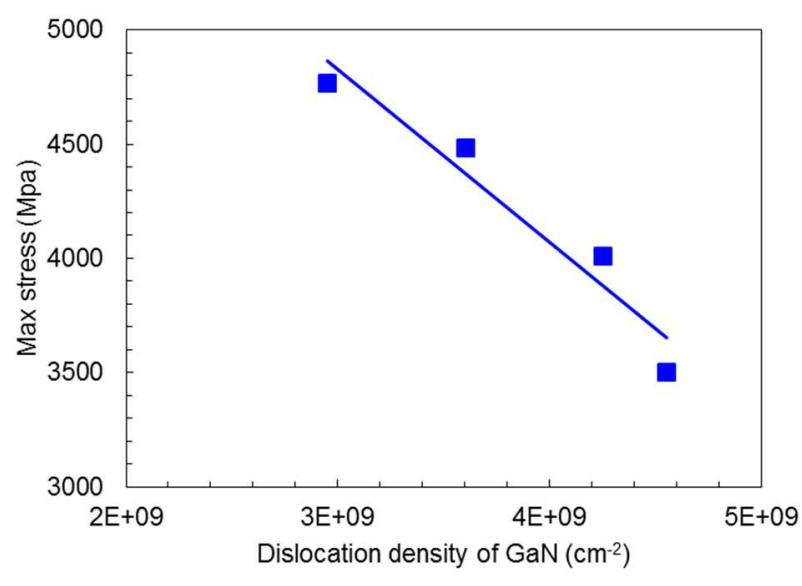

Figure 9: Peak stress versus dislocation density

\section{Conclusion}

In conclusion, we have shown that by appropriately treating the in-situ curvature data from $200 \mathrm{~mm} \mathrm{GaN}$ on silicon growth, we are able to extract the stress in the layers during growth. With this data, we are able to reconstruct XRD scans, showing good matching between measured values and reconstructed curves. The technique for fitting the slope of the in-situ curves also gives us a strong correlation between the maximum stress from the in-situ curvature plot and the density of dislocations in the layers, meaning that for these growth conditions, we can infer the dislocation density of the layers from the in-situ measurements.

\section{References}

[1] D. Christy, T. Egawa, Y. Yano, H. Tokunaga, H. Shimamura, Y. Yamaoka, A. Ubakata, T. Tabuchi and K. Matsumoto, "Uniform growth of AlGaN/GaN High Electron Mobility Transistors on 200mm silicon (111) substrate," Applied Physics Express, vol. 6, p. 026501, 2013.

[2] A.R.Boyd, S.Degroote, M. Leys, F. Schulte, O. Rockenfeller, M. Luenenbuerger, M. Germain, J. Kaeppeler and M. Heuken, "Growth of GaN/AlGaN on $200 \mathrm{~mm}$ diameter silicon (111) wafers by MOCVD," Physica Status Solidi C, vol. 6, pp. S1045-S1048, 2009.

[3] M. Charles, Y. Baines, S. Bos, R. Escoffier, G. Garnier, J. Kanyandekwe, J. Lebreton and W. Vandendaele, "The effect of AlN nucleation temperature on inverted pyramid defects in GaN layers growth on $200 \mathrm{~mm}$ silicon wafers," Journal of Crystal Growth, vol. 464, pp. 164-167, 2017.

[4] K. Cheng, H. Liang, M. V. Hove, K. Geens, B. D. Jaeger, P. Sreivastava, X. Kang, P. Favia, H. Bender, S. Decoutere, J. Dekoster, J. I. d. A. Borniquel, S. W. Jun and H. Chung, "AlGaN/GaN/AlGaN Double 
Heterostructures Grown on 200 mm Silicon (111)

substrates with high mobility," Applied Physics

Express, vol. 5, p. 011002, 2012.

[5] F. Semond, P. Lorenzini, N. Grandjean and J. Massies, "High electron mobility AlGaN/GaN heterostructure grown on $\mathrm{Si}(111)$ by molecular beam epitaxy," Applied Physics Letters, vol. 78, pp. 335337,2001

[6] K. A. Reinhardt and W. Kern, Handbook of Silicon Wafer Cleaning, Norwich, NY, USA: William Andrew Publishing, 2008, 2nd edition.

[7] M. Charles, Y. Baines, A. Bavard and R. Bouveyron, "High growth rate GaN on $200 \mathrm{~mm}$ silicon by metalorganic vapor phase epitaxy for high electron mobility transistors," Journal of Crystal Growth, vol. 483, pp. 89-93, 2018.

[8] F. Iacopi, M. V. Hove, M. Charles and K. Endo, "Power electronics with wide bandgap materials: Toward greener, more efficient technologies," MRS Bulletin, vol. 40, no. 5, pp. 390-395, 2015.

[9] G. Stoney, "The Tension of Metallic Films Deposited by Electrolysis," Proc. R. Soc. London, Ser. A, vol. 82, pp. 172-175, 1909.

[10] M. A. Hopcroft, W. D. Nix and T. W. Kenny, "What is the Young's Modulus of Silicon," Journal of Mircoelectromechanical systems, vol. 19, no. 2, pp. 229-238, 2010.

[11] H. Maruska and J. J. Tietjen, "The preparation and properties of vapor-deposited single crystal line GaN,” Applied Physics Letters, vol. 15, p. 327, 1969.

[12] G. A. Slack and S. F. Bartram, "Thermal expansion of some diamondlike crystals," Journal of Applied Physics, vol. 46, p. 89, 1975.

[13] W. M. Yim and R. J. Paff, "Thermal expansion of AlN, sapphire and silicon," Journal of Applied Physics, vol. 45, p. 1456, 1974.

[14] A. Polian, M. Grimsditch and I. Grzegory, "Elastic constants of gallium nitride," Journal of Applied Physics, vol. 79, p. 3343, 1996.

[15] L. E. McNeil, M. Grimsditch and R. H. French, "Vibrational Spectroscopy of Aluminium Nitride," Journal of the Americal Ceramic Society, vol. 76, no. 5, pp. 1132-1136, 1993. 